\title{
Determinación de Riesgos Existentes en la Industria Panificadora y la Manera de Evitarlos y Controlarlos
}

\section{Gustavo Efraín Carrera Oña, MsC, Investigador Vinicio Paredes Peralta, $\mathrm{MsC}$}

Docente de Tiempo Completo de la Facultad de Ciencias Pecuarias Escuela Superior Politécnica de Chimborazo (ESPOCH), Ecuador

\section{Juan Carlos Naranjo Herrera, MsC}

Docente de Tiempo Completo de la Facultad de Salud Public Escuela Superior Politécnica de Chimborazo (ESPOCH), Ecuador

\section{Darío Fernando Guamán Lozada, MsC Diego Iván Cajamarca Carrasco, MsC}

Docente de Tiempo Completo de la Facultad de Ciencias Pecuarias Escuela Superior Politécnica de Chimborazo (ESPOCH), Ecuador

Doi:10.19044/esj.2019.v15n13p19 ～URL:http://dx.doi.org/10.19044/esj.2019.v15n13p19

\section{Resumen}

En la presente investigación se realizó una evaluación de los riesgos laborales presentes en 6 panificadoras en la ciudad de Riobamba. El trabajo de la industria panificadora consiste en la elaboración de un alimento de tipo cereal como lo es el pan, el cual además de ser tradicional, se ha convertido en un producto de consumo diario. Esto hace de dicha industria una de las principales contribuyentes a la economía de un país o región. Las variables estudiadas en esta investigación en particular son los riesgos laborales, la señalización de materiales de protección, rutas de escape, además de posibles agentes externos como son las plagas y roedores. El objetivo del presente trabajo se logró a través de la encuesta pre-realizada al personal de las panificadoras, encuesta donde se determinaron los riesgos, los accidentes que estos implican y la manera de controlarlos. Posterior al estudio pre se ejecutó un plan de capacitación sobre los diferentes factores de riesgo, medidas preventivas, señalética de la panificadora y se llevó a cabo la elaboración del manual de prevención de riesgos laborales. Gracias a esto, fue posible concluir que hubo una disminución de riesgos y accidentes laborales en un $69 \%$. De igual manera se pudo contribuir a la promoción de una cultura de seguridad e higiene en las panificadoras a través de herramientas útiles para el empresario, esto con el fin de reducir los peligros y accidentes laborales y, de esta manera, manera mejorar la productividad y la calidad de sus productos. 
Palabras clave: Amenazas industriales, identificación de riesgos, recursos humanos, salud ocupacional, seguridad laboral

\title{
Determination of Existing Risks in the Bakery Industry and the Ways to Avoid and Control Them
}

\section{Gustavo Efraín Carrera Oña, MsC, Investigador Vinicio Paredes Peralta, $\mathrm{MsC}$}

Docente de Tiempo Completo de la Facultad de Ciencias Pecuarias Escuela Superior Politécnica de Chimborazo (ESPOCH), Ecuador Juan Carlos Naranjo Herrera, $\mathrm{MsC}$

Docente de Tiempo Completo de la Facultad de Salud Public Escuela Superior Politécnica de Chimborazo (ESPOCH), Ecuador

Darío Fernando Guamán Lozada, MsC Diego Iván Cajamarca Carrasco, $\mathrm{MsC}$

Docente de Tiempo Completo de la Facultad de Ciencias Pecuarias Escuela Superior Politécnica de Chimborazo (ESPOCH), Ecuador

\begin{abstract}
This paper focuses on evaluating the labor risks of 6 bakeries in the city of Riobamba. The work of the bakery industry consists of making a cerealtype food such as bread, which, in addition to being traditional, has become a product of daily consumption. This makes this industry one of the main contributors to the economy of a country or region. The variables studied in this research in particular are occupational hazards, signaling protection materials, escape routes, as well as possible external agents such as pests and rodents. The objective of the present work was achieved through the presurvey carried out on the personnel of the breadmakers, survey where the risks were determined, the accidents that these imply, and the way to control them. After the pre-study, a training plan was carried out on the different risk factors, preventive measures, and the signage of the bakery. In addition, the preparation of the manual for the prevention of occupational hazards was carried out. Thanks to this, it was possible to conclude that there was a decrease in risks and accidents at work by $69 \%$. In the same way, it was possible to contribute to the promotion of a safety and hygiene culture in bakeries through useful tools for the employer. This would help to reduce
\end{abstract}


occupational hazards and accidents and, in this way, improve productivity and the quality of their products.

Keywords: Industrial threats, risk identification, human resources, occupational health, job security

\section{INTRODUCCIÓN}

El pan es un cereal que apareció en la dieta del ser humano hace aproximadamente 14.400 A.C. (Arranz-Otaegui, Gonzalez Carretero, Ramsey, Fuller, \& Richter, 2018). Con la llegada de la Revolución Industrial en el siglo XVIII se produce un cambio en una sociedad cuando su economía deja de basarse en la agricultura y la artesanía para depender de la industria, industria que transforma la materia prima utilizando una fuente de energía obteniendo así productos elaborados o semielaborados. Esto trajo varias mejoras en los molinos, las cuales permitieron incrementar notablemente la producción de pan (Chaves Palacios, 2004).

A raíz de esto se desarrolla entonces la industria panificadora, uno de los grandes contribuyentes a la economía de un país o región al elaborar un alimento tan primordial y de consumo diario como lo es el pan. Esta industria ha tenido un crecimiento sostenido durante los últimos años, debido primordialmente a la gran acogida de los productos, además de a los programas emprendidos por el Gobierno Nacional del Ecuador que busca fortalecer el sector productivo del país (German Unda, 2014).

Como en toda industria, en la industria panificadora surge a lo largo de su crecimiento la necesidad de determinar los riesgos existentes y desarrollar un plan de seguridad apropiado para las mismas (Díaz, 2009).

En líneas generales, la seguridad en el trabajo es hqce referencia al conjunto de conocimientos técnicos y su aplicación para la reducción, control y eliminación de accidentes en el trabajo por medio de sus causas (Salgado Benítez, 2012).

Más específicamente, la seguridad industrial está dedicada a la protección de los trabajadores frente a accidentes, incendios, explosiones, exposiciones a sustancias tóxicas, vapores, gases, ruido entre otros, los cuales pueden estar expuestos los empleados (Chiavenato, 2006).

Los diversos accidentes que un trabajador puede sufrir se clasifican según el lugar donde ocurren (sucedidos en el lugar o centro de trabajo, yendo o viniendo del trabajo), según la situación laboral del trabajador (con baja o sin baja), y según la gravedad de la lesión a criterio médico (Marín, 2006). Además, es posible deducir una clasificación dependiendo de su origen las cuales pueden ser técnicas o humanas (Cortéz Díaz, 2005).

Cabe resaltar que con los numerosos accidentes de trabajo que han venido ocurriendo en la industria a lo largo de los años, en los países 
industrializados estos son considerados como un costo adicional de producción (Muñoz, 2004).

Para mitigar los accidentes laborales las industrias ofrecen medios de protección destinados exclusivamente a la protección del trabajador; es decir, a la eliminación o prevención del riesgo accidental (Cortéz Díaz, 2007). El objetivo principal del equipo de protección personal es servir como obstáculo entre el agente de riesgo, el cual puede ser un filo cortante, una superficie áspera, un objeto disparado o una sustancia peligrosa en el cuerpo del trabajador y así evitar lesiones que pueden producirse por el contacto con dichos agentes (Cortéz Díaz, 2005).

En este sentido, el presente artículo determinó los riesgos laborales latentes y la manera de controlarlos, en este caso orientado a panificadoras, e igualmente se observaron las causas más frecuentes de accidentes en los cuáles los empleados sufrieron las consecuencias.

Este documento obedece a la siguiente estructura: en el capítulo I, se aborda la Introducción y el planteamiento del problema. El capítulo II conlleva la metodología aplicada a la investigación; los resultados obtenidos son abordados en el capítulo III, en el capítulo IV se muestra la discusión de los resultados, en el capítulo $\mathrm{V}$ se visualizan las conclusiones y en el capítulo VI se aprecian las recomendaciones.

\section{OBJETIVOS}

\section{Objetivo General}

Determinar los riesgos existentes en la industria panificadora y la manera de evitarlos y controlarlos.

\section{Objetivos Específicos}

- Caracterizar la situación actual de las panificadoras y herramientas con las que cuenta para la producción.

- Identificar los factores que provocan los riesgos laborales e inseguridad en el trabajador.

- Aplicar alternativas de solución a la problemática analizada; capacitar al personal de las panificadoras y realizar un manual de prevención de riesgos laborales.

\section{METODOLOGÍA}

Se utilizó el método descriptivo en el que, mediante la observación, se relata el estado actual de las panificadoras. Adicionalmente, se observaron los posibles riesgos que estas presentan y con el método analítico se dedujo empíricamente cómo estas condiciones afectan a los trabajadores. También fue una investigación cuasi experimental en vista de la propuesta de un plan de prevención de riesgos laborales entre medio-trabajador; al implementar 
dicha propuesta se mejoraron notablemente las condiciones del ambiente de trabajo y se redujeron los accidentes en las panificadoras.

\section{○ Tipo de Estudio}

Tomando en cuenta el objeto de estudio esta investigación fue de tipo aplicada ya que se sustentó mediante la identificación de los riesgos y se le dio solución al problema a investigar con la propuesta del plan de prevención.

Por el lugar de donde se extrajeron los datos e informaciones pertinentes se puede decir que fue un estudio de campo. La investigación se ejecutó en 6 panificadoras de la ciudad de Riobamba, lugares donde se detectaron los riesgos.

En lo que respecta ql nivel del estudio, este fue expresivo y cuasiexperimental, ya que mediante el estudio del problema se establecieron las causas raíz de este y así se pudieron emitir soluciones.

\section{○ Técnicas}

Para recolectar la información se aplicaron encuestas con preguntas cerradas de opción múltiple y actas de recorrido en las que se registraron los riesgos y deficiencias que existen en las panificadoras por medio de matrices de riesgo.

\section{- Instrumentos de Evaluación}

Para el procesamiento de datos se utilizaron técnicas estadísticas a partir del software informático Microsoft Office Excel, mediante el cual se llegaron a establecer gráficos estadísticos a ser interpretados mediante el análisis y la inducción de datos, para así poder emitir conclusiones y recomendaciones.

\section{○ Análisis y Procesamiento de la Información (Procedimiento)}

1. Como primer paso se revisó bibliografía documental de fuentes secundarias para fundamentar la problemática y el objeto de estudio de la investigación.

2. Se identificó el campo donde se realizaría el estudio, el cual son 6 panificadoras en Ecuador localizadas en la provincia de Chimborazo, ciudad de Riobamba en las parroquias: Licto, Calpi, Maldonado, Velazco y Yaruquíes, en el periodo 2019-2020

3. Elaboración de encuestas pre para aplicar a los trabajadores y gerentes o propietarios de las panificadoras.

4. Se propuso, en base a los resultados de la investigación, un manual de seguridad y prevención de riesgos laborales para micro, pequeñas y medianas panificadoras.

5. Se procedió a la preparación del cuestionario de estudio Post, en donde 
se seleccionaron las mismas preguntas referentes al aspecto de la seguridad y prevención de riesgos laborales realizadas en el estudio Pre.

6. Para el análisis de los resultados se agrupó y tabuló la información para facilitar su análisis.

7. En la presentación de resultados se interpretó la información que se obtuvo de los cuestionarios realizados en las panificadoras apoyados en cuadros y gráficos estadísticos.

8. Para la interpretación de resultados, estos se compararon y confrontaron con lo que describe la teoría sobre los objetivos planteados.

9. Las conclusiones y recomendaciones se realizaron en base a cada objetivo planteado para dar solución a los mismos.

\section{RESULTADOS}

A continuación, se presentan los resultados de la entrevista al gerente o propietario de la panificadora. Dicha persona responde a la siguiente pregunta:

¿Cuántas personas laboran en el área operativa de la panificadora?

Tabla 1. Trabajadores de las panificadoras

\begin{tabular}{|c|c|c|}
\hline Panificadora & \# de Empleados & Total personas \\
\hline Panadería 1 & 5 & 6 \\
\hline Panadería 2 & 4 & 5 \\
\hline Panadería 3 & 5 & 6 \\
\hline Panadería 4 & 4 & 5 \\
\hline Panadería 5 & 5 & 6 \\
\hline Panadería 6 & 5 & 6 \\
\hline Total & 28 & 34 \\
\hline
\end{tabular}

Nota: Cabe resaltar que al total de personas de cada panificadora se suma uno porque el gerente o propietario se suma automáticamente y en todos los casos es uno.

\section{○ Resumen del Estudio Pre?}

Las encuestas se aplicaron a los empleados en cada uno de las panificadoras ya mencionadas en un horario no académico, incluyendo al gerente o propietario de las distintas panificadoras a las cuales se pudo acceder el viernes 30/11/2018 a las 17:30pm.

El personal encuestado manifestó a través de sus respuestas que la mayor parte de ellos sufre accidentes laborales como se observa en la Figura 1: 


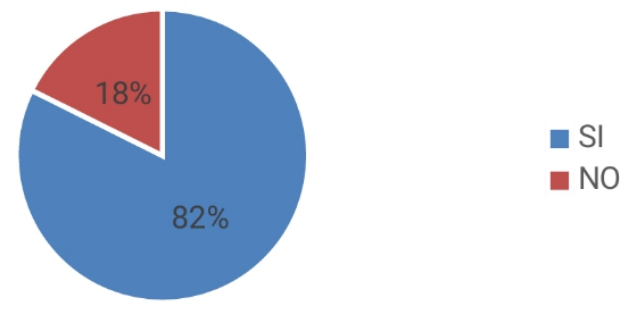

Figura 1. Accidentes de trabajo

Al realizar la encuesta al personal, estos manifestaron que los accidentes laborales se deben a la falta de conocimiento en factores de riesgo y seguridad por parte de gran parte del personal (ver Tabla 2). Aquellos que los conocen lo hacen ya que por su experiencia y antigüedad fueron obligados a aprender por sí solos. Además, las panificadoras no cuentan con un plan o manual de prevención de riesgos en el que el $100 \%$ de los empleados respondieron que no y el $0 \%$ sí. (Ver Tabla 3).

Tabla 2. Conocimiento de factores de riesgo laboral

\begin{tabular}{|c|c|}
\hline Denominación & Frecuencia \\
\hline SI & $11 \%$ \\
\hline NO & $89 \%$ \\
\hline Total & $100 \%$ \\
\hline
\end{tabular}

Tabla 3. Existencia de plan de seguridad y prevención de factores de riesgo

\begin{tabular}{|c|c|}
\hline Denominación & Frecuencia \\
\hline SI & $0 \%$ \\
\hline NO & $100 \%$ \\
\hline Total & $100 \%$ \\
\hline
\end{tabular}

La ausencia de protección con equipos adecuados es otro de los mayores problemas que presentaron las panificadoras, ya que si bien la mayoría del personal utiliza diversos equipos para protegerse (mandiles, batas y cofias), algunos de ellos presentan deterioro y no son todos los equipos que en verdad deberían usarse. Las mascarillas son adquiridas por cuenta propia del personal y no son provistas por parte de la empresa y el equipo faltante (mascarillas y guantes térmicos) no es brindado a loes empleados (Figura 2):

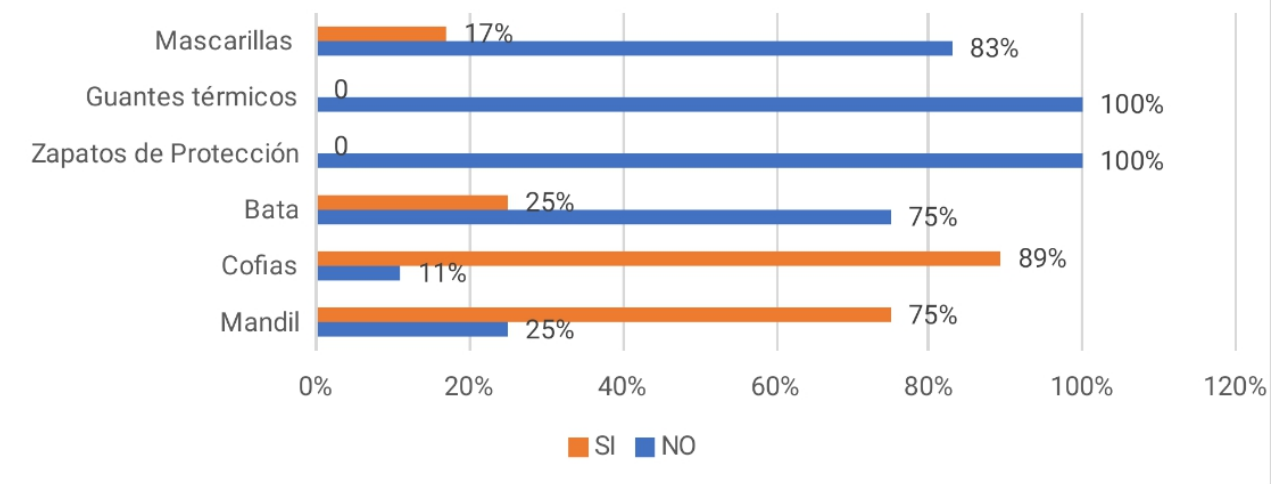

Figura 2. Uso de equipo de protección personal 
Por la falta del equipo de protección personal ya mencionado se observó que un gran porcentaje del personal tuvo accidentes,entre ellos: laceraciones, quemaduras, cortes, caídas, tropiezos, entre otras como se puede observar en la Figura 3. Es importante mencionar que el personal que no ha sufrido accidentes son nuevos o provisionales en su área y no llevaban mucho tiempo ejerciendo.

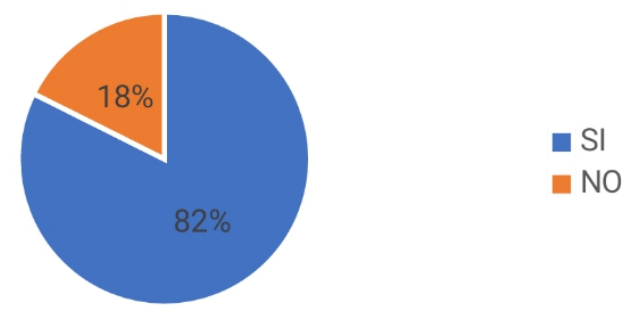

Figura 3. Accidentes de trabajo en personal

Cabe recalcar que el ambiente de trabajo no cuenta con la higiene adecuada, esto debido al derrame de desechos líquidos y grasos en el piso ya que no llevan un control de orden y limpieza, razón por la cual en algunas panificadoras existe la presencia de plagas. No obstante, todas mantienen un control especialmente sobre hormigas, ratones y cucarachas, pero este no está automatizado al $100 \%$ ya que el control se realiza manualmente a través de matamoscas, insecticidas, trampas para ratones, venenos, entre otros.

Se observó igualmente que no se encuentran identificados ni rotulados los materiales de producción, maquinarias, herramientas y utensilios de trabajo pues los propietarios asumen que los empleados tienen conocimiento de ellos. El área de producción y los equipos no están diseñados ni son los adecuados para una secuencia de procesos de producción. Las señales más conocidas por los empleados son de precaución, información y prohibición, sin embargo, las panificadoras no cuentan con la señalización requerida dentro de sus instalaciones. Por la falta de señalización se han ocasionado algunos accidentes que no han sido graves, pero que han retrasado la productividad de ciertas panificadoras.

- Plan de capacitación y elaboración del manual de prevención de riesgos laborales en las panificadoras de la ciudad de Riobamba.

En base a los problemas identificados y mediante la investigación en base al objeto de estudio, se propuso un plan de capacitación estructurado, y un manual de prevención de riesgos laborales. 


\section{○ Objetivos del Plan de Capacitación}

- Capacitar al personal de las panificadoras para que conozcan los diferentes factores de riesgo y el impacto que estos tienen en el mejoramiento del proceso laboral.

- Incentivar al gerente o propietario de las panificadoras a hacer uso del manual de prevención de riesgos, siento este un recurso de vital importancia en el ejercicio panificador.

\section{- Resumen del Estudio Post}

Una vez aplicada la encuesta post al grupo en experimento se obtuvieron los siguientes resultados:

Al realizar la encuesta al personal, estos manifestaron que con los conocimientos de los riesgos laborales estos tendrán más cuidado al momento de producción (ver Tabla 4).. Además, ya todas las panificadoras cuentan con un plan o manual de prevención de riesgos (Ver Tabla 5).

\begin{tabular}{|c|c|}
$\begin{array}{c}\text { Tabla 4. Conocimiento en facto } \\
\text { de riesgo laboral }\end{array}$ \\
\hline Denominación & Frecuencia \\
\hline SI & $100 \%$ \\
\hline NO & $0 \%$ \\
\hline Total & $100 \%$ \\
\hline
\end{tabular}

Tabla 5. Existencia de plan de seguridad y prevención de factores de riesgo

\begin{tabular}{|c|c|}
\hline Denominación & Frecuencia \\
\hline SI & $100 \%$ \\
\hline NO & $0 \%$ \\
\hline Total & $100 \%$ \\
\hline
\end{tabular}

Tomando en cuenta los resultados obtenidos podemos afirmar que existe un impacto significante en los trabajadores ya que al conocer los riesgos laborales estos podrán ejercer su profesión con más cautela y así mejorar su producción.

La ausencia de protección no es un problema ya que con los equipos adecuados la gerencia o propietario de la panificadora tomó cartas en el asunto en lo que respecta a cuidar a sus trabajadores para reducir sus riesgos y evitar accidentes. Esto queda demostrado en el hecho de que los equipos proporcionados ya no presentan deterioro pues son nuevos y el equipo faltante como los guantes térmicos, zapatos de protección y mascarillas ya son provistos igualmente por la empresa (Figura 4):

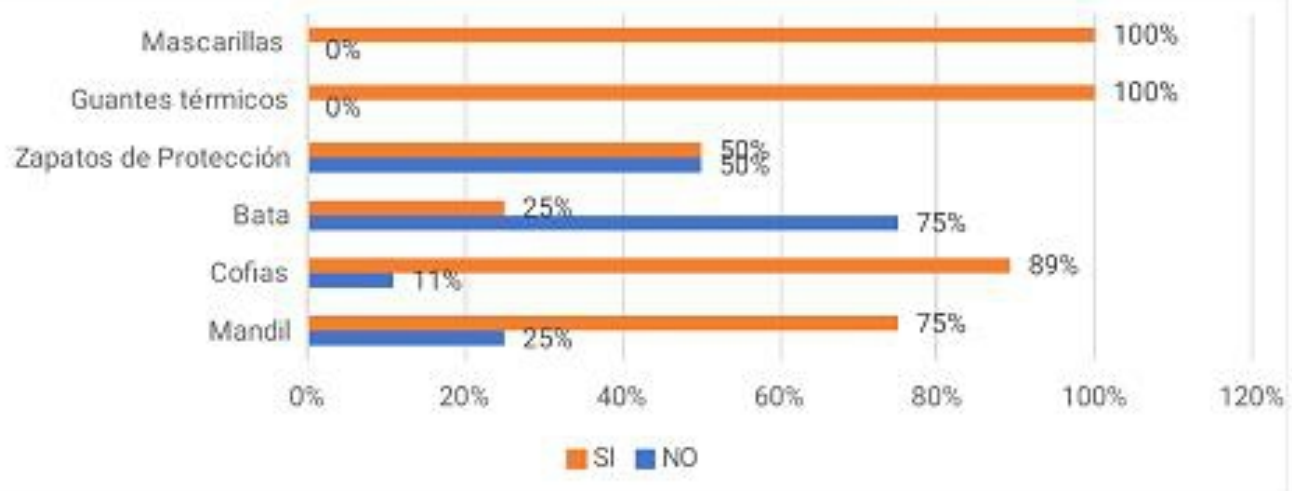

Figura 4. Uso de equipo de protección personal 
Nota: Es evidente que casi todos los empleados prefieren usar mandil en vez de bata. Los zapatos de protección fueron provistos mayormente al personal de amasado y horneado pues, al ser el equipo más costoso, tomará más tiempo poder proporcionarlo a todos los empleados, según manifestó el gerente o propietario de las panificadoras faltantes en proveer dicho equipo de protección.

El ambiente de trabajo mejoró considerablemente ya que cuenta con la higiene adecuada gracias a que se lleva un orden y limpieza. Esto conllevó a la la ausencia considerable de plagas, especialmente hormigas y cucarachas.

Se observó que en la planta ya se encuentra identificados y rotulados los materiales de producción, maquinarias, herramientas y utensilios de trabajo, además de que los empleados en un $100 \%$ conocen las señales dentro de la instalación y los materiales ya mencionados.

Con todas las precauciones de riesgos ya mencionados el personal manifestó que hubo un cambio notable para evitar accidentes aumentando su satisfacción y seguridad laboral. La gran mayoría de los empleados no ha tenido ningún accidente laboral, y en el caso de aquellos que sí lo han tenido estos no han sido graves: tropezones, caídas o cortes leves. Los empleados que tuvieron dichos accidentes están conscientes que han sido por negligencia propia (Figura 5).

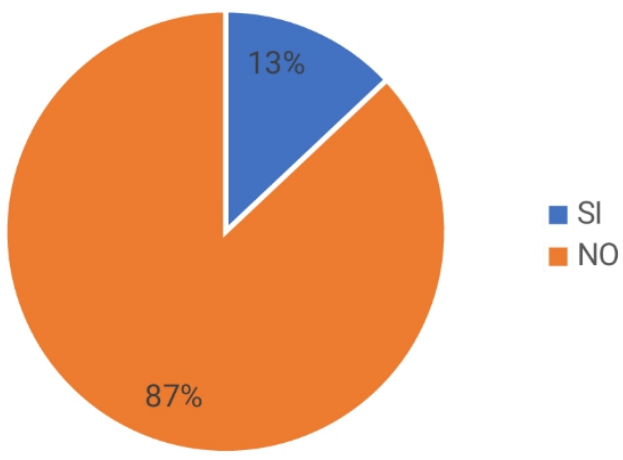

Figura 5. Accidentes Laborales

\section{- Mapa de riesgos}

A continuación, en la Figura 6 se muestra una matriz de riesgos desde diferentes ángulos, y que según la matriz de riesgos insht conocemos con exactitud los lugares donde se corre más riesgo en diferentes situaciones ya sean químicas físicas biológicas etc., al momento de trabajar en dicho lugar. 


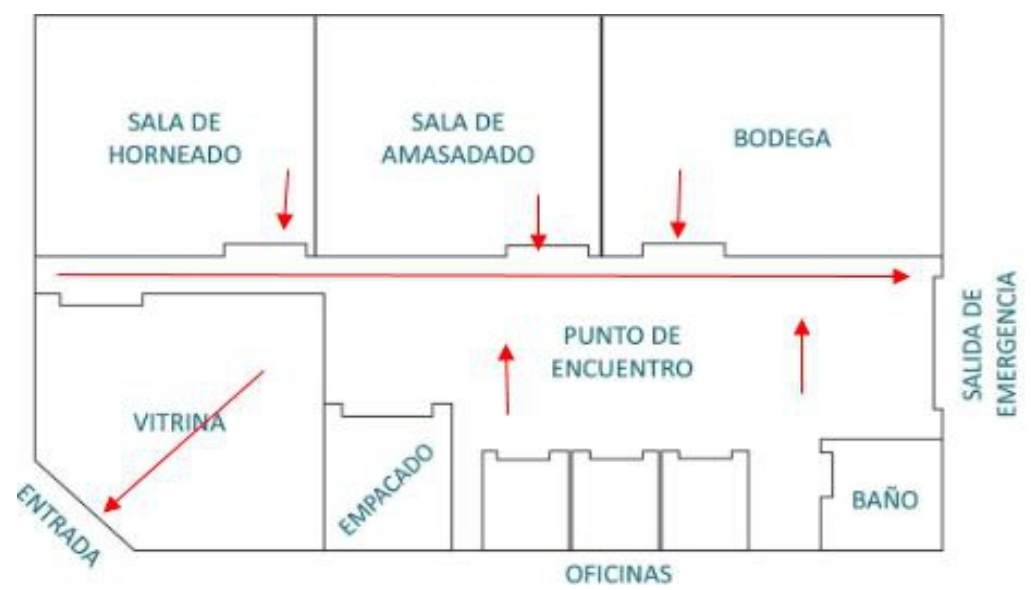

Figura 6. Mapa de matriz de riesgos panadería Fuente: (Díaz, 2009)

En la Figura 7 se dan a conocer las áreas de peligro dentro de la panadería para así tratar de reducir los riesgos o accidentes de trabajo dentro de estas zonas que se muestran a continuación:

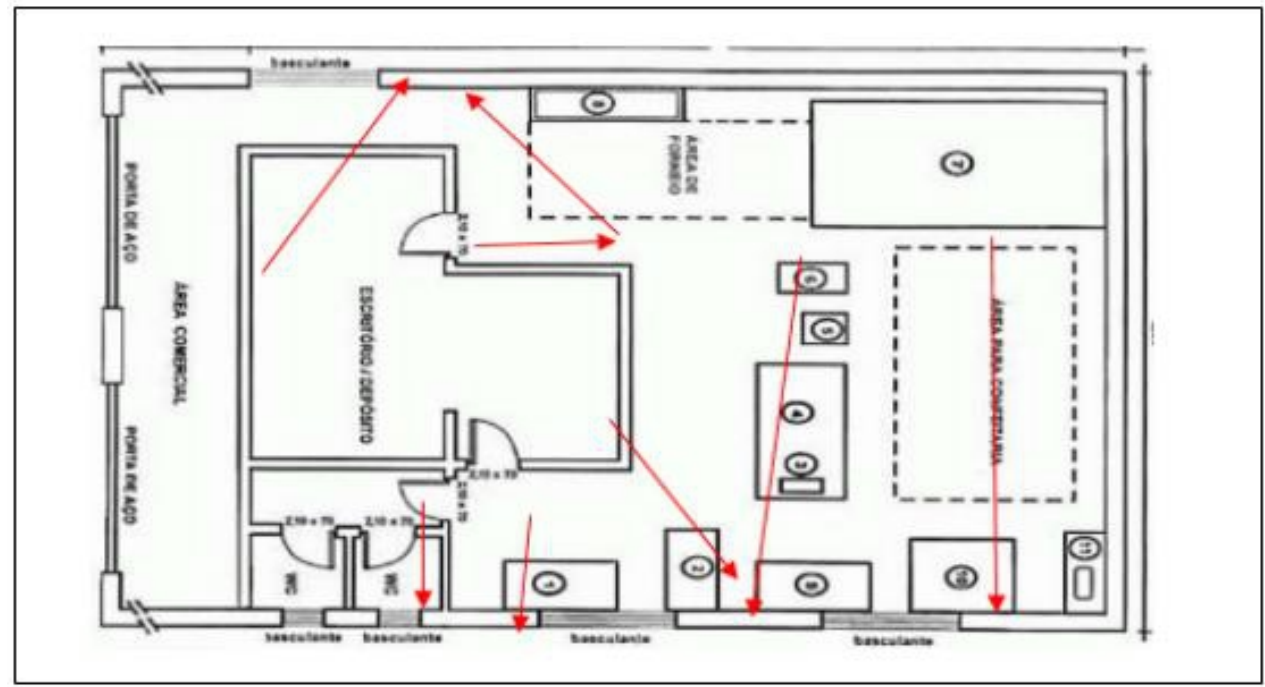

Figura 7. Mapa de matriz de riesgos panadería

Fuente: (Cortés, J., 2007)

En la Figura 8 se muestran las áreas de mayor riesgo dentro del control, producción, transporte, y almacenamiento de los productos de la panadería, áreas de trabajo en las cuales hay que tener sumo cuidado para evitar accidentes laborales que puedan traer graves consecuencias. 


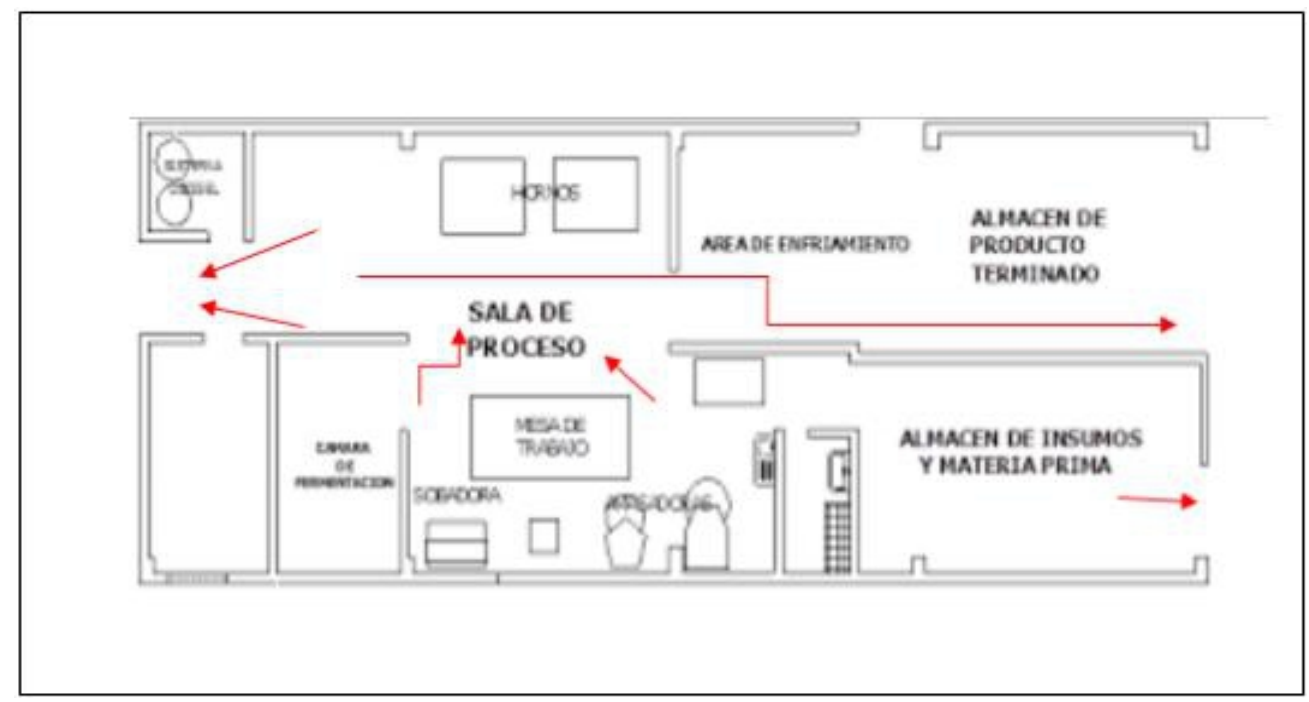

Figura 8. Mapa de matriz de riesgos panadería Fuente: (Díaz, 2009).

En la Figura 9 se aprecian diferentes zonas de circulación de los trabajadores en las cuales puede haber un mayor índice de riesgos laborales debido al tráfico constante y movilidad de productos, por lo tanto se necesita un mejor control de dichas zonas.

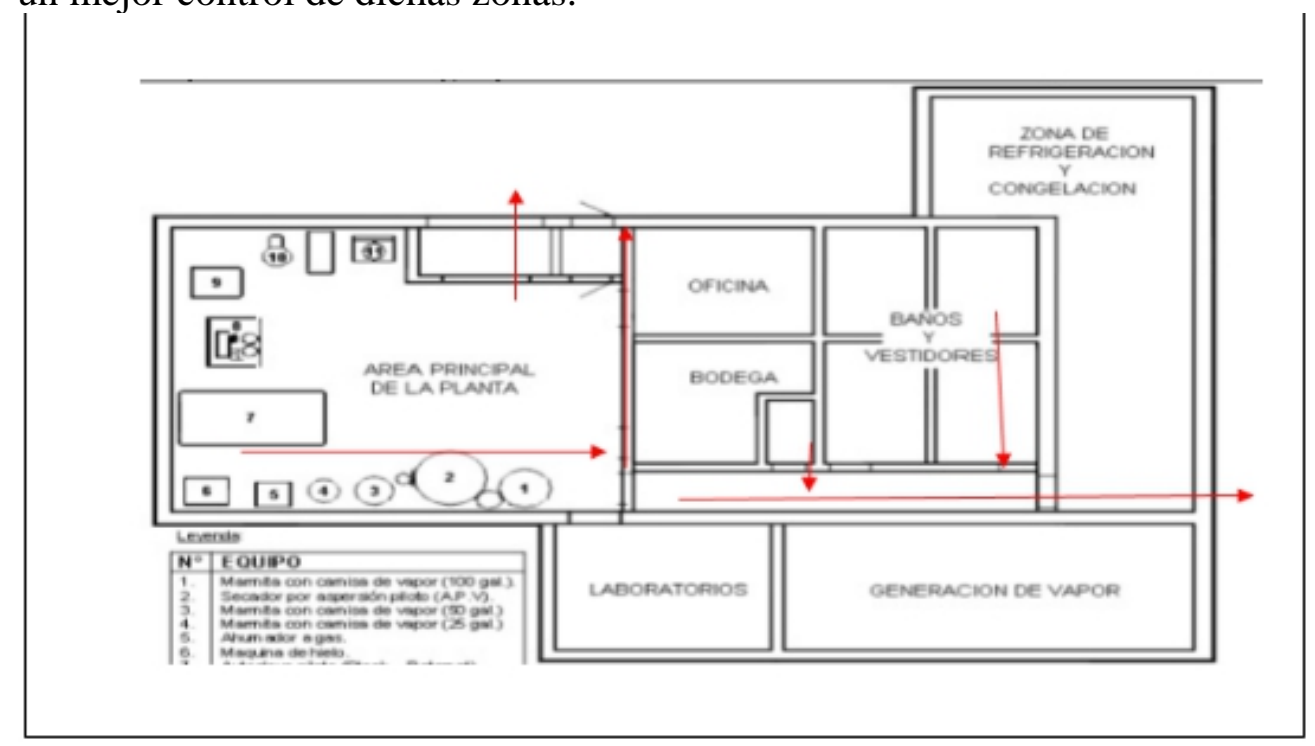

Figura 91. Mapa de matriz de riesgos panadería

Fuente: (Espeso, 2009) 


\section{DISCUSIÓN DE RESULTADOS}

Al hablar de seguridad industrial para es posible afirmar que la seguridad en el trabajo busca la prevención de accidentes y administra los riesgos ocupacionales. Se anticipa, a efecto de que los riesgos de accidentes sean mínimos (Chiavenato I. , 2009) . En otras palabras, la seguridad industrial está dedicada en un 95\% a la protección de los trabajadores, ante la pérdida que podrían sufrir por accidentes, incendios, explosiones, exposiciones a sustancias tóxicas, vapores, gases, ruido, entre otros, sea cual sea el tamaño de la industria.

El total del personal administrativo entrevistado en el presente estudio indicó que en las panificadoras no se cuenta con un manual de prevención de accidentes, justificando que no ha sido necesario o por considerarse una empresa pequeña. Por tanto, existe desconocimiento y falta de interés de los propietarios o administradores por brindar seguridad a sus empleados.

Dentro de la industria estudiada, se determina que los accidentes ocurridos con más frecuencia suelen ser las quemaduras, resbalones y tropiezos, con una ocurrencia entre una o dos veces al año. Tanto el personal operativo como administrativo convergen en que la mayor consecuencia de los accidentes sufridos, debido al tipo actividad, son lesiones físicas, siendo las áreas del cuerpo más afectadas manos, brazos, pies y piernas (Saari, Jørgensen, Raouf, Skiba, \& Andreoni ).

El personal administrativo no posee un registro de los accidentes ocurridos, y d carecen de manuales que permitan dar seguimiento a las acciones para contrarrestar las consecuencias de los incidentes; por lo tanto, se hace evidente la necesidad de crear una guía preventiva acerca del tratamiento de accidentes y primeros auxilios, además de realizar un análisis y registro de accidentes para detectar puntos críticos que los ocasionen y eliminarlos (Saari, Jørgensen, Raouf, Skiba, \& Andreoni ).

Cortéz Díaz (2007) establece que la protección del personal tiene como objetivo proteger al trabajador frente a agresiones externas ya sean de tipo físico, químico o biológico. En la investigación se comprueba que no existe una cultura de prevención y seguridad personal; los empleados operativos consideran necesarios para realizar su labor únicamente la gabacha y gorra como protección, mientras que los propietarios reconocen que el equipo de protección personal reduce o elimina las lesiones que pueda sufrir el trabajador. En el área de producción de las panificadoras es necesario que los colaboradores utilicen gabachas, zapatos con suela antideslizante, cofias o redecillas, lentes transparentes, guantes térmicos y mascarillas.

Uno de los factores con más influencia en la prevención de accidentes es el orden y limpieza de los locales de trabajo, siendo la misión de la señalización dar información a través de un conjunto de estímulos (Cortez Díaz, 2007). Las panificadoras no poseen un programa de señalización, orden 
y limpieza, pero las instalaciones de producción, en su mayoría, se encuentran limpias y ordenadas. Sin embargo, el 95\% de los materiales de producción, áreas de trabajo, maquinaria, herramientas y utensilios de trabajo no se encuentran identificados y rotulados, pues consideran que los empleados los reconocen. Al presentar tanto al personal operativo como administrativo una tabla de señalizaciones, los empleados indican tener más percepción de las señales de prohibición, precaución e información, siendo las de forma gráfica las más identificadas debido a los colores, pictogramas y leyendas o combinación de éstos (Martínez, 2016).

Referente al ambiente del trabajo, (Hernández Zúñiga, 2003) señala que existen diversos factores que pueden desfavorecer el área de trabajo, los cuales afirma corresponden a las condiciones de: lugar de trabajo, maquinaria, equipo, herramientas, materias primas, procesos de fabricación, espacios; además de actos donde se originan los contaminantes que pueden ser: polvo, humo, gases, ruido, calor etc. En las empresas es importante destacar que la concentración de vapor y humo producido por el combustible es constante en estos espacios provocando un escenario desfavorable para los empleados del área de producción. El 33\% de las panificadoras que fueron objeto de este estudio no mantiene alejados los combustibles de los focos de calor, lo cual puede provocar incendios y explosiones poniendo en peligro la seguridad de los empleados.

En lo que respecta a la ergonomía, (Gonzalez Maestre, 2007) denota que el objetivo de este elemento es la prevención de daños en la salud física, mental y social mediante la interrelación hombre-artefacto o colaboradormaquinaria. En la empresa el personal administrativo considera que la ubicación de la maquinaria de trabajo es idónea, y las sillas, bancos, mesas y clavijeros son adecuados y se encuentran en buen estado.

Otro aspecto fundamental para este estudio se relaciona con la higiene industrial, cuyo objetivo es la prevención de enfermedades profesionales y del trabajo causadas por contaminantes tanto físicos, químicos y/o biológicos, generados en el ambiente de trabajo (Mercader Uguina , 2007). En la investigación se encontró que el total de las panificadoras sujeto de estudio no cuentan con una guía de prevención de salud.

Dentro de las enfermedades profesionales relacionadas con la panificación se encuentran los resfriados en un $47 \%$ debido a los cambios de temperatura repentina, ya que las áreas de producción son cálidas y al salir de allí se encuentran con temperaturas heladas o corrientes de aire frío; así mismo están las ocasionadas por agentes psicológicos y físicos como el cansancio ya que el $68 \%$, presentan dolores musculares y estrés, debido al tipo de trabajo que realizan (Universidad Privada Telesup, 2014). 


\section{CONCLUSION}

- Dentro de la industria estudiada, se determina que los accidentes ocurridos con más frecuencia suelen ser las quemaduras, resbalones y tropiezos, con una ocurrencia entre una o dos veces al año..

- El personal administrativo no posee un registro de los accidentes ocurridos, además carecen de manuales que permitan dar seguimiento a las acciones para contrarrestar las consecuencias de los incidentes, evidenciando así la importancia de crear una guía preventiva para las empresas correspondientes. Se determinó que no existe una cultura de prevención y seguridad personal, pues si bien los propietarios están conscientes de la importancia del equipo de protección personal para reducir accidentes, los empleados operativos consideran necesarios para realizar su labor sólo algunos de los equipos de protección (gabacha y gorra).

- El 33\% de panificadoras no mantiene alejados los combustibles de los focos de calor, lo cual puede provocar incendios y explosiones poniendo en peligro la seguridad de los empleados; además no cuentan con guías de prevención. Por lo tanto, es indispensable que las panificadoras implementen un Manual de seguridad y salud, y que tengan un registro para tener antecedentes y poder dar el seguimiento adecuado en todas las situaciones de enfermedades o riesgos laborales.

- La señalización de las áreas y materiales de trabajo tuvo un efecto positivo en lo que respecta a la correcta identificación de los espacios por parte de los empleados.

- Luego de la implementación del manual de seguridad propuesto el porcentaje de accidentes ocurridos en las panificadoras estudiadas fue bajo.

\section{RECOMENDACIONES}

1. Además de los riesgos externos a los trabajadores estos deben cuidarse por sí mismos, razón por la cual deben tomar en cuenta su correcta postura para proteger su espalda, tomar descansos regulares, evitar laborar en estado etílico y tratar de reducir el estrés laboral.

2. Cada trabajador tiene todo el derecho de informar al supervisor o gerente acerca de cualquier peligro de seguridad en el lugar de trabajo.

3. Tomar las precauciones debidas en el uso de herramientas y no tomar "atajos" laborales ya que esta puede ser una causa de lesión.

4. Cada empleado tiene que ser consciente de su entorno ya que debe aprender sobre los riesgos potencialmente peligrosos y mantenerse separado de estas áreas o posibles situaciones peligrosas.

5. Los administradores o gerentes de las panificadoras deben mantener un acceso rápido y fácil a la salida de emergencia de además de 
mantener un acceso claro al cierre de ciertas maquinarias en caso de una emergencia para que paren rápidamente su funcionamiento.

6. Replicar el manual de prevención de riesgos laborales a todas las pequeñas y medianas panificadoras para disminuir, evitar y controlar los riesgos y contribuir al mejoramiento de la industria.

\section{References:}

1. Arranz-Otaegui, A., Gonzalez Carretero, L., Ramsey, M. N., Fuller, D. Q., \& Richter, T. (2018). La evidencia arqueobotánica revela los orígenes del pan hace 14,400 años en el noreste de Jordania. Proceedings of the National Academic Science (PNAS), 6.

2. Chaves Palacios, J. (2004). Desarrollo tecnológico en la Primera Revolución Industrial. Norba. Revista de historia, 93-109.

3. Chiavenato, I. (2006). Administración de recursos humanos: fundamentos básicos. Sao Paulo: Atlas.

4. Chiavenato, I. (2009). Administración de recursos humanos. Río de Janeiro: Elsevier Ltda.

5. Cortés, J. (2007). Seguridad e Higiene del trabajo. Madrid : Tebar SL.

6. Cortéz Díaz, J. M. (2005). Salud y seguridad en el trabajo. Costa Rica: EUNED.

7. Cortéz Díaz, J. M. (2007). Seguridad e higiene del trabajo. Madrid: Tebar SL.

8. Díaz, M. (2009). Salud y Seguridad en trabajos de minería. Buenos Aires: Aulas y Andamios.

9. Espeso, J. E. (2009). Seguridad en el trabajo manual para la formación del especialista. España: Lex Nova, S. A.

10. German Unda, D. A. (Diciembre de 2014). Diseño de un sistema de gestión por procesos y propuesta de mejora en una empresa panificadora ubicada en la ciudad de Quito. Caso: Panificadora Mi Buen Pan. Quito, Pichincha, Ecuador.

11. Gonzalez Maestre, D. (2007). Ergonomía y psicología. Madrid: Fund. Confemetal.

12. Hernández Zúñiga, A. (2003). Seguridad e Higiene industrial. Mexico: Limusa.

13. Marín, F. (2006). Seguridad industrial manual para la formación de ingenieros. . Madrid: Dykinson.

14. Martínez, M. (12 de Julio de 2016). Preventiam. Obtenido de Preventiam: https://preventiam.com/top-10-consejos-seguridadtrabajo/

15. Mercader Uguina, J. R. (2007). Mutuas de accidentes de trabajo y enfermedades profesionales. Madrir: Las Rozas (Madrid); La Ley. 
16. Muñoz, J. (2004). La gestión integrada: calidad, seguridad y medio ambiente. . España: SERFOREM, S.L.

17. Saari, J., Jørgensen, K., Raouf, A., Skiba, R., \& Andreoni , D. (s.f.). PREVENCION DE ACCIDENTES. ACCIDENTES Y GESTION DE LA SEGURIDAD, 46.

18. Salgado Benítez, J. (2012). Higiene y seguridad industrial. México: Exodo.

19. tematico8. (s.f.). asturias.es. Obtenido de asturias.es: https://tematico8.asturias.es/export/sites/default/consumo/seguridadA limentaria/seguridad-alimentariadocumentos/BUENAS_PRxCTICAS_HIGIxNICAS.pdf

20. Universidad Privada Telesup. (2014). Seguridad y Salud Ocupacional. Peru. 